\title{
A Case Study on Time-Interval Fuzzy Cognitive Maps in a Complex Organization
}

\section{Minzoni, Angela}

\section{IEEE}

2017

Minzoni , A , Mounoud , E \& Niskanen , V A 2017 , A Case Study on Time-Interval Fuzzy Cognitive Maps in a Complex Organization . in 2017 8th IEEE International Conference on Cognitive Infocommunications (CogInfoCom) . International Conference on Cognitive Infocommunications, IEEE , pp. 27-32, 8th IEEE International Conference on Cognitive Infocommunications (CogInfoCom), Debrecen , Hungary , 11/09/2017 . https://doi.org/10.1109/CoglnfoCom.2017.8?

http://hdl.handle.net/10138/312786

https://doi.org/10.1109/CogInfoCom.2017.8268211

unspecified

acceptedVersion

Downloaded from Helda, University of Helsinki institutional repository.

This is an electronic reprint of the original article.

This reprint may differ from the original in pagination and typographic detail.

Please cite the original version. 

Debrecen, Hungary

\section{A Case Study on Time-Interval Fuzzy Cognitive Maps in a Complex Organization}

\author{
Angela Minzoni \\ Eléonore Mounoud \\ Centrale Supelec \\ Laboratoire Génie Industriel \\ Université Paris-Saclay \\ Châtenay-Malabry, France \\ Angela.minzoni@ecp.fr, Eléonore.mounoud@ecp.fr
}

\author{
Vesa A. Niskanen \\ Dept. of Economics \& Management \\ University of Helsinki \\ Helsinki, Finland \\ vesa.a.niskanen@helsinki.fi
}

\begin{abstract}
Temporal issues within modeling organizational systems are examined generally and with fuzzy cognitive maps. These maps give the opportunity to consider temporal factors when studying organizational models. The knowledge we gain about the system is useful when the aim is not to optimize time intervals in well-known and instrumented contexts, but also to discover the behavior of the system while different temporal factors are implemented by the management. We will present an adapted resolution for including these factors as key elements in organizational models with fuzzy cognitive map examples for middle and back office application.
\end{abstract}

Keywords - temporality, organizational design, coordination, fuzzy cognitive maps

\section{INTRODUCTION}

When modelling organizational and social systems, it is important to acknowledge the importance of temporal aspects, both in duration and pace, because some relationships not only base on the immediate causal interconnections but there may be a certain time interval between the causes and their effects. The identification of temporality in interrelationships are particularly challenging in model constructions.

Minzoni and Mounoud provide in [7] an overview of the general methodological approach adopted for the system under study, viz., the middle and back office operations for banking services. It should to be recalled here that the operations are considered as embedded in their organizational context, i.e., including strategy, governance, procedures, etc. It is thus the behavior of the entire system which is considered, knowing that the available data are almost exclusively qualitative and that there are no tools giving a global picture of the necessary interconnections to carry out the operations. In our study fuzzy cognitive maps (FCM) have provided a method for experts to elicit and identify interrelationships between the given concepts for understanding the features of the system when temporal factors are introduced in the model. Thus, this is one approach to the complex mixture of human and artificial cognitive capabilities in human computer interaction processes as well as to socio-cognitive ICT which utilizes or influences on collective knowledge [24]. The data and expertise presented below are thus originating from real back office operations.

Our interrelationships are only approximately stated, weak (0.5) or strong (1), positive or negative, because our principal aim is not to support decision making but rather to provide more knowledge about the organizational system and the global view by uncovering previously unknown patterns to the management team. FCMs enable us to simulate temporal scenarios and to understand system's global operational states by providing holistic information rather than to optimize its parts to the detriment of the whole.

The FCMs thus seem to be viable models for examining the foregoing systems because they enable us to study the complex phenomena of the real world $[1,6,8,11,14]$. Their constructions usually base on human expertise or empirical (historic) data. Both numeric and linguistic models may be applied in this context $[4,6]$. However, the extended time inclusive approach is still quite rarely adopted within the FCMs even though it plays an important role particularly when organizational design aspects are involved in these systems $[4,18,21]$.

This study introduces temporal approach to FCM by representing relations that are not always active in a simulation. This method allows a direct modeling of real world situations that are not much currently addressed in FCM. Chapter 2 considers how temporal aspects have been considered within organization studies. Chapter 3 considers the challenges of FCM construction especially when time intervals are involved in these models, and detailed examples are provided. These considerations are based on the FCM models and their computer simulations carried out by Niskanen, following the initiatives and monitoring of Minzoni, and at the first stage, also by the work of the Hungarian research group under the supervision of Koczy [2,19]. Chapter 4 concludes our considerations.

\section{CONSIDERING TEMPORAL FACTORS IN ORGANIZATIONS}

Our study pivots on the problems of causality and temporality. In causality we may apply causa efficiens and causa finalis if Aristotle's terms are used. The former is used in the 
Natural Sciences, whereas both of these are applied to the Human Sciences (this also including Economics).

The final causes seem more challenging to dynamical systems because they represent teleological relationships which are typical of the human goal-oriented behavior, i.e., their present acts are the effects caused by their goals located in the future. Also, unlike in the control applications based upon the deterministic efficient causes, the socio-economic systems often deal with relationships which are only probabilistic by nature. Various causal chains are also more usual and thus our systems may include delays and other temporal factors.

When trying to understand the large corporations' dynamic organization, the temporal issue is a key concept. This issue is mostly considered by introducing "delay" that means time spans considered too long comparatively to the expected time. In order to estimate delays, the system needs to be understood well enough for knowing what normal and delayed time intervals and durations are. Not all systems have already such a refined quantitative knowledge to estimate whether their cause-effect relations are delayed or immediate. When the behavioral pattern of the system is unknown, application of temporal factors in FCM calculations allows experts to discover this pattern, throwing new light on their system's understanding and time span calibration [10,18,20-22].

We can picture time in different ways according to cultures and organizations, drawing on different sets of imagery, or using different metaphors [5]. Anthropologists have often described cultures as having either cyclical or linear notions of time. Cyclical time, naturally enough, emphasizes repetition and is very much influenced by the cycles of the natural world. The line and the cycle are the similar two key time metaphors by which time is thought in organization theory. In the history of organization, the focus has initially been upon linear time images that stem from the progressive commodification of the labor process. An example is the homogeneous time-reckoning systems of Taylorism [16]. Taylor maintained time as a commodity to be factored into job design, organization processes and control mechanisms.

Hassard [5] has pointed out beyond the issues of clocktime, the importance of what has been referred to as "instantaneous-time", whereby organizational practices are based on time-frames that lie beyond conscious human experience. $\mathrm{He}$ associates this concept with the complex shifts from Fordism to the flexible accumulation of "post-Fordism". Management and organization theory have viewed time as a significant resource and put forward multiple views as to how time is both managed and experienced [3]. The way we experience time is open to manipulation and specifically time compression that displaces critical reflection and may make individuals "managed by (others') time". In [12] was developed the idea of the speed trap and showed how the need for fast action, traditionally conceptualized as an exogenous feature of the surrounding context, is a product of an organization's own past emphasis on speed.

More recently in [13] was studied the influence of clocktime orientation market-based models for human development. While a linear, clock-time orientation optimized for markets is meant to enhance efficiency, coordination, and control, it may be unsuitable for managing emergent, complex, and indeterminate processes such as development. This work develops an agentic view of time, where time is used as a cultural resource to regulate attention and render social phenomena amenable to managerial action and how organizations accommodate seemingly contradictory temporal orientations.

In this study, we used FCM modeling to establish a collective (and thus possible to share and discuss between organizational members) overview on the interrelationships and other temporal issues, such as effects of speed, fire-fighting, escalation and fast pace course of action [12]. FCMs were used to support the discovery of organizational hidden patterns. In our case this means the temporal patterns for providing ways of integrating each person's personal schema as well as the backdrop of the organization's objectives. It helps both to interpret the information and to coordinate that information to make it meaningful for the members of the organization and its goals. It enables the sense-making process by which organizational members incessantly create and re-create conceptions of themselves and of the world all around them. As described in [17], sense-making is an action which involves turning circumstances into a situation that is comprehended explicitly in words or speech and that serves as a springboard to action. In [18], in turn, was noticed that research and theory on organizations are lacking a critical specification on time scale. Specification of the relevant time scale is as critical as the specification of the appropriate level or unit of analysis, a concept to which it is related. They showed how the choice of time scale has important implications for the development of organization theory.

After unfolding the simulation details by applying temporal factors in FCM, we will present a short reflection from the organizational science perspective. Within this context organizational patterns are understood as those internal relationships which provide the system with its coherence and keep it from being an accumulation of fragmented activities.

\section{Model CONSTRUCTION EXAMPLES ON TEMPORAL FuZZY COGNITIVE MAPS}

In our project, Niskanen, Koczy and Hatwagner carried out thorough analysis of expert-based FCM models which represented the middle and back office operations for banking services in a private French bank (and thus certain confidentiality should be maintained below) [19].

Standard methods were used in basic simulations, and thus the concept (node) values ranged from zero to unity and the weights or intensities of the interrelationships in the connection matrices belonged to the closed intervals from -1 to $1[6,11,14]$. Our FCM models contained 12 to 35 concepts, each of these being possible inputs and ouputs, and expectedly, especially the large connection matrices were particularly challenging in computations due to their many parameters.

Hence, in the basic FCM computer simulations we applied the matrix product, *,

$$
\mathrm{V}_{\mathrm{t}+1}=f\left(\mathrm{~V}_{\mathrm{t}} * \mathrm{M}\right)
$$

in which state vector, $\mathrm{V}_{\mathrm{t}}$, contains $\mathrm{m}$ concept values at time $\mathrm{t}, \mathrm{M}$ is an $\mathrm{m} \times \mathrm{m}$ connection matrix, $f$ is the transformation function and vector $\mathrm{V}_{\mathrm{t}+1}$ contains the new concept values at time 
$\mathrm{t}+1$ [11]. One time unit of $\mathrm{t}$ denoted in our models one month. For function $f$ we applied the usual sigmoid function,

$$
f(\mathrm{x})=1 /(1+\exp (-\lambda \mathrm{x})),
$$

in which $\exp$ is the exponential function and parameter $\lambda>0$ [11].

Numerous simulations were performed when our models were studied [19]. First 1000 to 10000 random initial concept vectors were used for each FCM model for examining their general variation in iterations. In this context, various lambda values were also tested for obtaining their optimal values, i.e., the values which caused large standard deviations to the output values [19]. In this manner, we also observed such variation as possible fixed-point attractors and examined statistical distributions of concept outcomes in our models.

Various alternative initial state vectors and connection matrices were also tested by using genetic and bacterial evolutionary algorithms in optimization. By assigning appropriate goal vectors, this method revealed us how fixed-point attractors or chaotic variation can be created, inter alia. We also examined how to reduce the converging speed of the state vectors to their fixed points.

Below we focus on the above-mentioned temporal issues. We aimed to also apply such external temporal factors which could take into account the distinct time intervals between the causes and their effects in our FCM modeling. This approach is thus a more challenging task than with those prevailing models which are based on historic data or do not deal with the extended temporal aspects $[4,11,21]$.

Our methods for temporal factors seem somewhat analogous to those of in $[20,22]$ from the theoretical standpoint, but in practice we used different calculations in the models. Hence, this paper provides a case study based on a real-world application.

According to the foregoing simulations, we used lambda value of 1 because then the outputs have larger dispersions in their transformed values even though value of 5 seems more frequent within the FCMs. The value of 1 is also used below.

Since lack of space precludes wider analyses, we only focus below on a concrete example concerning our bank's organizational FCM with its thirteen concepts, viz.,

$1=$ clients, $2=$ rules $\&$ regulations, $3=$ new IT solutions, $4=$ funding, $5=$ cost reduction, $6=$ profit $/$ loss, $7=$ investments, $8=$ staff, $9=$ new services, $10=$ quality, $11=$ client development, $12=$ service development, 13 = productivity.

In the real-world FCM applications the preceding values of concepts are usually relevant in the succeeding iteration. Thus, in our computations the diagonals of our connection matrices also contained nonzero values, and we mainly used the values of 1 .

Table 1 provides an example on a connection matrix which was specified according to bank's experts (Fig. 1). One challenge in simulations was that the matrices only contained a quite small number of rounded weights which is a typical feature, and disadvantage, in expert-based models. Thus, with this matrix, our concept values may vary as presented in Tables 2 and 3 when 1000 random initial concept vectors are used. These statistics are only approximate by nature because, due to the combinatorial explosion, we were unable to use all combinations of values as initial vectors.

We notice in Table 2 that the ranges of some concepts values are so wide that it is justified to use small values of $\boldsymbol{\lambda}$ in (2) because then the corresponding transformation function is not too steep. However, Table 3 presents, for example, that still small concept values are not obtained. In addition, our basic FCM models often yielded quite fast fixed-point attractors to our concept values (Fig. 2). In this case the fixed-point attractors were virtually attained in less than ten iterations. Most of the concepts also have values very close to the maximum of 1 . However, these results still seemed to allow the managers to acknowledge the non-chaotic variation of their organization.

Since we were unable to influence on the values of weights in computation, we do not consider below much the meaningfulness of our concepts or their interrelationships but rather we focus on their mere technical and computational aspects in order to acquire information by examining their patterns of behavior and variation. Matlab ${ }^{\mathrm{TM}}$ and $\mathrm{R}$ with their Toolboxes were used in computations.

TABLE 1. EXAMPLE OF OUR CONNECTION MATRIX, M.

\begin{tabular}{|c|c|c|c|c|c|c|c|c|c|c|c|c|c|}
\hline & 1 & 2 & 3 & 4 & 5 & 6 & 7 & 8 & & 10 & 11 & 12 & 13 \\
\hline 1 & 1 & 0 & 0.5 & 0 & 0 & 0.5 & 1 & 0.5 & 0 & 0.5 & 1 & 0.5 & \\
\hline 2 & 1 & 1 & 0.5 & 1 & 0 & & 1 & 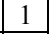 & 0.5 & 0 & & 1 & \\
\hline 3 & 1 & 0.5 & 1 & 0 & & & & -1 & 1 & 0 & & 1 & \\
\hline 4 & 0 & 0 & 0 & 1 & 0 & & & & 0 & 0 & 0 & 0 & \\
\hline 5 & 0 & 0 & 1 & -0.5 & 1 & & & -1 & 0 & 0 & 0 & 1 & \\
\hline 6 & 0 & & & & & & & & & & & & \\
\hline 7 & 0.5 & 0 & 0.5 & & & & & & 0 & -0.5 & 0 & 0 & \\
\hline 8 & \begin{tabular}{|l|}
0 \\
\end{tabular} & 0 & 0 & 0 & & -0.5 & & & 0 & 0.5 & 0 & 0 & -0. \\
\hline 9 & \begin{tabular}{|l|}
0 \\
\end{tabular} & 0 & & 1 & & 0.5 & 0. & 0.5 & 1 & -0.5 & 0 & 0.5 & \\
\hline 10 & 0.5 & 0 & 0 & 0 & & 0 & 0.5 & 0.5 & 0.5 & 1 & 1 & 0 & \\
\hline 11 & \begin{tabular}{|l|}
0 \\
\end{tabular} & 0 & 0.5 & 0. & & & 0 & \begin{tabular}{|l|} 
\\
\end{tabular} & 0.5 & 0.5 & \begin{tabular}{|l|}
1 \\
\end{tabular} & 0 & \\
\hline 1 & 0 & 0 & 0.5 & 0.5 & & & & & 0.5 & 0 & 0.5 & 1 & -0. \\
\hline & 0 & 0 & 1 & 0 & & & & & & & & & \\
\hline
\end{tabular}

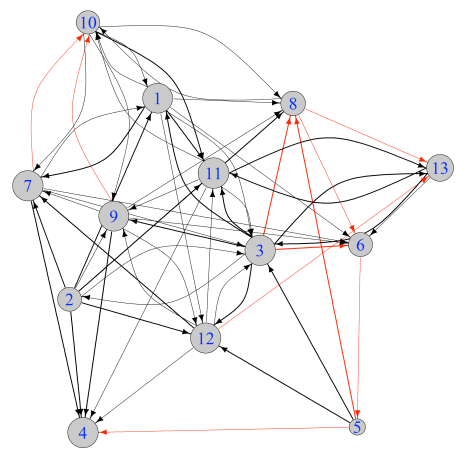

Fig. 1. Fuzzy cognitive map based on Table 1 (self-loops are not presented).

Moreover, generally in the human-scientific models, we should have both causal and teleological temporal interconnections. The latter factors, which comprise peoples' intentions, motives and their other underlying causes, are more challenging than the causal effects. Thus, often in these real-world ap- 
plications, the FCM models should also take into account the time spans, delayed effects and even the present goals of the decision makers, and these aspects have not been studied much thus far $[4,10,18,20,21-23]$.

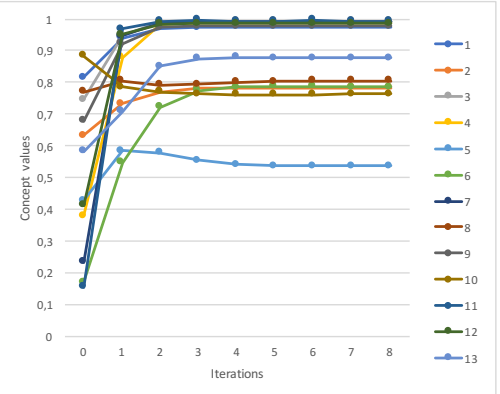

Fig. 2. Concept values in eight iterations when applying method (1), iteration 0 denotes random initial values.

Table 2. Possible Concept Values By Using Our Connection Matrix PRIOR TO TRANSFORMATION OF (2).

$\begin{array}{lccccc} & \text { Range } & \text { Min } & \text { Max } & \text { Mean } & \text { Std. Deviation } \\ \text { Clients } & 4 & 0 & 4 & 2.01 & 0.55 \\ \text { Rules_regulations } & 1.5 & 0 & 1.5 & 0.77 & 0.32 \\ \text { NewITsolutions } & 5.5 & 0 & 5.5 & 2.76 & 0.62 \\ \text { Funding } & 4.5 & 0 & 4.5 & 2.25 & 0.67 \\ \text { Cost_reduction } & 1.46 & -0.46 & 1 & 0.26 & 0.32 \\ \text { Profit_loss } & 2.96 & -0.86 & 2.09 & 0.71 & 0.52 \\ \text { Investments } & 5 & 0 & 5 & 2.48 & 0.64 \\ \text { Staff } & 3.83 & -1.26 & 2.58 & 0.73 & 0.64 \\ \text { New_services } & 4 & 0 & 4 & 2.00 & 0.52 \\ \text { Quality } & 2.51 & -0.53 & 1.99 & 0.76 & 0.43 \\ \text { Client_development } & 6.5 & 0 & 6.5 & 3.27 & 0.74 \\ \text { Service_development } & 5 & 0 & 5 & 2.49 & 0.63 \\ \text { Productivity } & 3.32 & -0.59 & 2.72 & 1.03 & 0.54\end{array}$

TABle 3. Possible Concept Values by Using Our Connection MatriX AFTER TRANSFORMATION (2).

$\begin{array}{lccccc} & \begin{array}{c}\text { Range } \\ \text { Clients }\end{array} & \text { Min } & \text { Max } & \text { Mean } & \begin{array}{c}\text { Std. Devia- } \\ \text { tion }\end{array} \\ \text { Rules_regulations } & 0.48 & 0.5 & 0.98 & 0.87 & 0.06 \\ \text { NewITsolutions } & 0.32 & 0.5 & 0.82 & 0.68 & 0.07 \\ \text { Funding } & 0.5 & 0.5 & 1 & 0.93 & 0.04 \\ \text { Cost_reduction } & 0.49 & 0.5 & 0.99 & 0.89 & 0.06 \\ \text { Profit_loss } & 0.33 & 0.39 & 0.72 & 0.56 & 0.08 \\ \text { Investments } & 0.59 & 0.3 & 0.89 & 0.66 & 0.11 \\ \text { Staff } & 0.49 & 0.5 & 0.99 & 0.91 & 0.05 \\ \text { New_services } & 0.71 & 0.22 & 0.93 & 0.66 & 0.13 \\ \text { Quality } & 0.48 & 0.5 & 0.98 & 0.87 & 0.06 \\ \text { Client_development } & 0.51 & 0.37 & 0.88 & 0.68 & 0.09 \\ \text { Service_development } & 0.5 & 0.5 & 1 & 0.95 & 0.04 \\ \text { Productivity } & 0.49 & 0.5 & 0.99 & 0.91 & 0.05 \\ & 0.58 & 0.36 & 0.94 & 0.73 & 0.10\end{array}$

For example, political decisions often have impacts in the long term, whereas in control applications immediate responses are usual. One well-known resolution to temporal factors is suggested in [18] who, all in all, applied nonlinear, conditional and fixed time-delayed relationships. In [10] fixed time delays were also used. In [23] simple cause-effect, time-delay causal and conditional probabilistic relations, as well as sequential, special time-delayed, relations were suggested. These extended FCM models based on their manual developments, and thus more or less subjective values to the delays were also assigned. In [21] a genetic learning algorithm method with data, referred to as the higher order FCM, was introduced which was able to take into account the given number of the previous concept values when their current values were computed.

As stated above, we also considered temporal factors in our framework. In $[20,22]$ three types of meanings for delay were presented, time delay between the cause and effect, the period of time when the cause is actualizing in the effect, and the combination of these two. This approach to temporal aspects was somewhat analogous to our objectives below. Hence, we assumed in the simulations first that different time intervals may prevail between the causes and their effects, and second, that there are even different time intervals or periods of time for the process how certain causes will emerge. In practice, in our extended temporal models certain nonzero links were always active, whereas others were inactive except in the given iterations. A simple real-world example would be the quarterly changes of Central Bank's interest rates to the markets because they stay fixed or inactive in certain iterations.

The use of time intervals and other additional temporal factors opens new prospects for examining the FCM models. For example, then the concept values may start oscillating, and this type of variation often corresponds better to the daily variation between busy and more relaxed time periods in the company or organization.

We used our own matrix-based calculation methods in computing because this approach may be more user-friendly when time intervals are specified and simpler in computations. Thus, we designed first an additional $\mathrm{m} \times \mathrm{m}$ matrix, $\mathrm{D}$, which controlled the actions of the concepts (Table 4). This matrix represented the time intervals within the impacts of the concepts. Hence, if the link from concept $C_{i}$ to $C_{j}$ is only active in every $k^{\text {th }}$ iteration, then $D(i, j)=k$. If link $C_{i}$ to $C_{j}$ is always active, $D(i, j)=1$. Otherwise $D(i, j)=0$ when $i$ and $j=1,2,3, \ldots$, $\mathrm{m}$. In computations, in each iteration, $\mathrm{k}$, we created with $\mathrm{D}$ such $\mathrm{m} \times \mathrm{m}$ indicator matrix, $\operatorname{IM}_{\mathrm{k}}$, in which $\operatorname{IM}_{\mathrm{k}}(\mathrm{i}, \mathrm{j})=1$ when $\mathrm{D}(\mathrm{i}, \mathrm{j})=\mathrm{k}$ or 1 , otherwise $\mathrm{IM}_{\mathrm{k}}(\mathrm{i}, \mathrm{j})=0$. We now replaced in $(1)$ the operation $\mathrm{V}_{\mathrm{t}}{ }^{*} \mathrm{M}$ by $\mathrm{V}_{\mathrm{t}}{ }^{*}\left(\mathrm{M} \cdot \mathrm{IM}_{\mathrm{k}}\right)$ in which $\mathrm{M} \cdot \mathrm{IM}_{\mathrm{k}}$ means the cell-by-cell multiplication of $\mathrm{M}$ and $\mathrm{IM}_{\mathrm{k}}$. Hence, in iterations $\mathrm{k}$ $\geq 1$,

$$
\begin{aligned}
\mathrm{V}_{\mathrm{t}+1}= & f\left(\mathrm{~V}_{\mathrm{t}} *\left(\mathrm{M} \cdot \mathrm{IM}_{\mathrm{k}}\right)\right) \\
& \text { TABLE 4. EXAMPLe OF OUR Matrix, D. }
\end{aligned}
$$

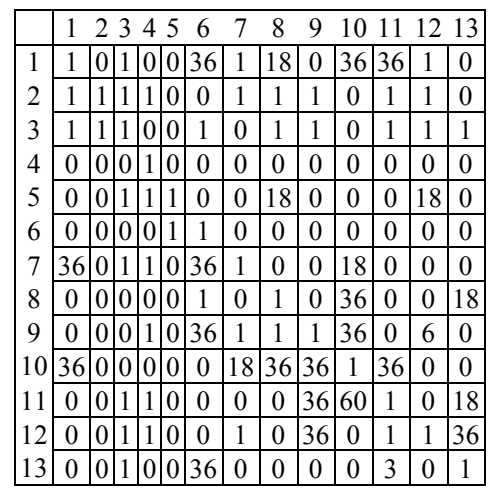

We also applied the $\mathrm{m} \times \mathrm{m}$ frequency matrix, $\mathrm{F}$, which controlled the frequencies of taking action in the occasional 
links in matrix D (Table 5). Hence, $\mathrm{F}(\mathrm{i}, \mathrm{j})>1$ when $\mathrm{D}(\mathrm{i}, \mathrm{j})>1$, otherwise $F(i, j)=0$. Now the actual initial values for the time intervals were $\mathrm{D}=\mathrm{D}+\mathrm{F}$. In each iteration, $\mathrm{k}>1$, we applied method (3) and then updated those cell values of D in which $\mathrm{D}(\mathrm{i}, \mathrm{j})=\mathrm{k}$,

$$
D(i, j)=D(i, j)+F(i, j) ; i, j=1,2, \ldots, m \text {. }
$$

For example, if originally $\mathrm{D}(\mathrm{i}, \mathrm{j})=6$ months and $\mathrm{F}(\mathrm{i}, \mathrm{j})=3$ months, then first $\operatorname{IM}_{k}(i, j)=1$ (and the link $M(i, j)$ is thus active) in iteration $k=6+3=12$. After this, $M(i, j)$ is again active when $\mathrm{k}=9+\mathrm{n} \cdot 3$ and $\mathrm{n}=2,3, \ldots$

TABLE 5. EXAMPLE OF OUR MATRIX, F.

\begin{tabular}{|c|c|c|c|c|c|c|c|c|c|c|}
\hline & & & & & & & & & & \\
\hline & \begin{tabular}{|l|l|l}
0 & 0 & 0 \\
\end{tabular} & 0 & 0 & $\begin{array}{l}3 \\
\end{array}$ & 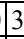 & & 3 & & & \\
\hline & \begin{tabular}{|l|l|l|}
0 & 0 & 0 \\
\end{tabular} & & 0 & \begin{tabular}{l|l}
0 & 0 \\
\end{tabular} & & & & & & \\
\hline & \begin{tabular}{|l|l|l}
0 & 0 & 0 \\
\end{tabular} & 0 & 0 & & 0 & & & & & \\
\hline & \begin{tabular}{|l|l|l}
0 & 0 & 0 \\
\end{tabular} & & 0 & & & & & & & \\
\hline & \begin{tabular}{|l|l|l|}
0 & 0 & 0 \\
\end{tabular} & 0 & 0 & & & 10 & & & & \\
\hline & & & 0 & & 0 & & & & & \\
\hline & \begin{tabular}{|l|l|l}
3 & 0 & 0 \\
\end{tabular} & 00 & 0 & & 0 & 0 & & & & 0 \\
\hline & \begin{tabular}{|l|l|l}
0 & 0 & 0 \\
\end{tabular} & 00 & 0 & & & & & & & \\
\hline & \begin{tabular}{|l|l|l}
0 & 0 & 0 \\
\end{tabular} & 00 & 0 & & & & & & & \\
\hline & \begin{tabular}{|l|l|l}
3 & 0 & 0 \\
\end{tabular} & & 0 & & & & & & & 0 \\
\hline & \begin{tabular}{|l|l|l|}
0 & 0 & 0 \\
\end{tabular} & & 0 & & & & & & & \\
\hline & \begin{tabular}{|l|l|l|}
0 & 0 & 0 \\
\end{tabular} & & 0 & & & & & & & \\
\hline & & & & & & & & & & \\
\hline
\end{tabular}

Fig. 3 depicts in a simplified form this variation of the profit/loss concept when only the link from investment has the time interval of 6 and frequency of 3 months. Hence, this link is first active in $6+3=9^{\text {th }}$ iteration, and after this in every $3^{\text {th }}$ iteration. We notice that instead of converging to the fixed point, the profit/loss concept now has more limit-cycling variation. Introducing time through time intervals and frequencies provides a different viewpoint on the system. It enables the system to oscillate. This variation is consistent with the daily experience of ups and downs, the alternation of fast pace and more relaxed periods in the company.

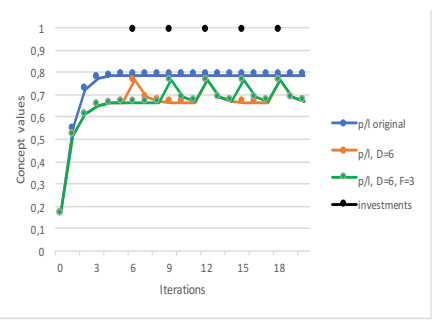

Fig. 3. Variation of profit/loss concept values with method (3) when investments have time interval of 6 and frequency of 3 months (iteration 0 denotes random initial values).

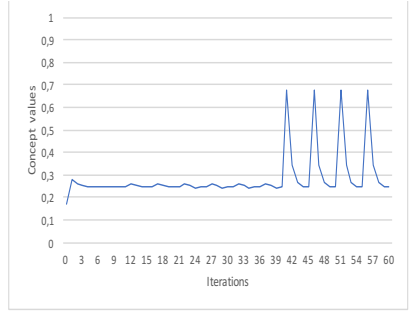

Fig. 4. Example on the variation of profit/loss concept with method (3) when time intervals and frequencies in Tables 4-5 are used (iteration 0 denotes random initial values).
Fig. 5 depicts this variation with all our concepts together. We notice limit-cycling, or even chaotic, variation compared to Fig. 2, as was expected in these conditions. Table 6 presents the possible concept values within 60 iterations when 1000 random initial concept vectors were used. We notice somewhat more variation in the concept values than in Table 3 , but still small values are not obtained.

Also, depending upon the values of frequencies and time intervals, the amplitude of oscillations can change dramatically, and for example Fig. 4 illustrates well an idea on the abovementioned speed trap in [12]. It reveals the impacts of the activating links with higher frequency than desired and the types of their outcomes. We thus may observe how the crests and troughs, traditionally conceptualized as an exogenous feature of the surrounding context, are a consequence of frequently activating links and thus being a result of organizational behavior. In the manner of ECG curve revealing the functioning of the heart, Fig. 4 is revealing in the transactions system the changing values of our concepts. We may thus diagnose if any 'tachycardia' is affecting on the system.

Table 6. Possible Concept Values When Matrices D AND F ARE Also USED IN 60 ITERATIONS.

$\begin{array}{lccccc} & \text { Range } & \text { Min } & \text { Max } & \text { Mean } & \text { Std. Deviation } \\ \text { Client } & 0.47 & 0.5 & 0.97 & 0.94 & 0.02 \\ \text { Rules_regulations } & 0.32 & 0.5 & 0.82 & 0.78 & 0.02 \\ \text { NewITsolution } & 0.5 & 0.5 & 1 & 0.99 & 0.01 \\ \text { Funding } & 0.49 & 0.5 & 0.99 & 0.99 & 0.02 \\ \text { Cost_reduction } & 0.34 & 0.39 & 0.72 & 0.61 & 0.02 \\ \text { Profit_loss } & 0.51 & 0.2 & 0.72 & 0.32 & 0.15 \\ \text { Investments } & 0.49 & 0.5 & 0.99 & 0.98 & 0.01 \\ \text { Staff } & 0.6 & 0.29 & 0.9 & 0.73 & 0.03 \\ \text { New_services } & 0.47 & 0.5 & 0.97 & 0.91 & 0.03 \\ \text { Quality } & 0.23 & 0.5 & 0.73 & 0.64 & 0.04 \\ \text { Client_development } & 0.5 & 0.5 & 1 & 0.97 & 0.02 \\ \text { Service_development } & 0.49 & 0.5 & 0.99 & 0.97 & 0.02 \\ \text { Productivity } & 0.42 & 0.5 & 0.92 & 0.87 & 0.03\end{array}$

Fig. 5 also provides an overview of the system and shows the multiple oscillations that create crests and troughs in the model. It highlights the importance to coordinate frequencies in relation to time intervals for avoiding (uncomfortable when experienced) huge variations in too many domains in the organization.

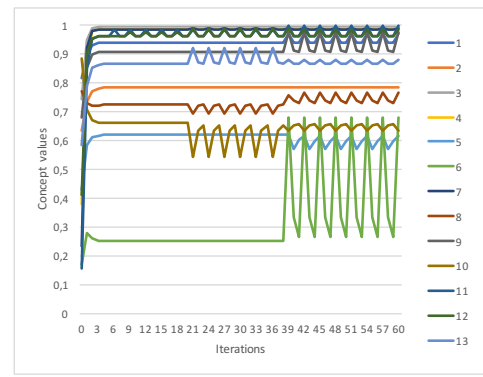

Fig. 5. Concept values when also time intervals and frequencies in Tables 4-5 are used (iteration 0 denotes random initial values).

The numeric FCM approach which we have adopted thus far is not the only possibility to operate with temporal aspects $[4,18]$. In the numeric case, we will meet additional challenges due to their mathematical properties [18]. One central challenge to us was how we can optimize the connection matrix weights from the mathematical standpoint if historic data is unavailable? The human experts can only assign a more or less limited number of subjective and rounded values, and this may naturally affect on the goodness of the outcomes. This question still arises even though certain resolutions have been 
suggested already [20,21]. This was one study area in which we also tested with evolutionary computing such alternative connection matrices which yielded lower concept values or attained with reduced speed the fixed-point attractors [19]. Various values of parameter $\boldsymbol{\lambda}$ were also tested in this context. We examined each tentative model with 1000 random initial vectors for finding their essential characteristics [19].

\section{CONCLUSIONS}

In the last decade, management sciences have become increasingly interested in and influenced by operations research, in particular intensive data acquisition and computing capabilities. The organizations nevertheless encounter such situations in which, prior to operationally optimizing their existing practices, they instead of only performing these operations in a faster or cheaper manner, have to consider new ways of organizing operations within broader conceptual frameworks.

When having this type of wide problem area, the available data are highly heterogeneous and we often lack appropriate historic data. In such cases FCMs may still provide a particularly suitable method when elaborating macro models for delivering such provisional assumptions. These models, in turn, help the Managers to keep a prospective attitude while running their daily operations.

There may also be various time intervals between the causes and their effects, and these temporal factors in interrelationships are particularly challenging in model construction. This issue was studied in the light of FCM modeling above. Since only a few studies have examined this type of temporal aspects, one new method was suggested and used in our applications. It applied both time intervals and their frequencies, and a concrete example was presented. Thus, we could identify which impulses were sent too frequently, and this enabled us to calibrate managerial actions and objectives in necessariy time rather than disrupting the system by over-sending impulses.

Our temporal effects caused more variation to the model's concept values, as was expected. Another interesting feature was that the systems could oscillate and reveal different oscillation amplitudes for concept value's, while keeping their stability. This oscillatory variation may be compared to the ECGtype results in cardiology.

Our FCM modeling thus seems to shed new light for organizing and monitoring such operations which comprise long time intervals and presuppose the project members to respond in good time and with full understanding of the organization's dynamics. Such time patterns contribute to reflexive practices within the organization, saving energy and preventing stress. More generally, such socio-cognitive ICT and computational intelligence was applied which both utilized and influenced upon collective knowledge in an organization.

Our innovative results address the capital issue of introducing explicit temporalities in the FCM. However, the computerization of time intervals in the complex dynamical systems is still a quite a new frontier, and thus further studies are expected in this area.

\section{ACKNOWLEDGEMENTS}

We express our thanks to the anonymous referees for their valuable comments.

\section{REFERENCES}

[1] R. Axelrod, Structure of Decision, The Cognitive Maps of Political Elites. Princeton University Press, Princeton, 1976.

[2] M. Buruzs, M. Hatwágner and L. T. Kóczy, "Expert-Based Method of Integrated Waste Management Systems for Developing Fuzzy Cognitive Map”, in Q. Zhu and A. Azar (Eds.), Complex System Modelling and Control Through Intelligent Soft Computations. Studies in Fuzziness and Soft Computing, Vol. 319, pp. 111-137, 2015.

[3] A. Carr, and Ph. Hancock, "Space and time in organizational change management", Journal of Organizational Change Management, Vol. 19 Iss: 5 , pp. $545-557,1988$.

[4] J. P. Carvalho and J. Tome, "Rule Based Fuzzy Cognitive Maps in Socio-Economic Systems", Proceedings of the IFSA Congress, pp. 1821-1826, Lisbon, 2009.

[5] J. Hassard, "Commodification, construction and compression: a review of time metaphors in organizational analysis", pp. 131-140, Version of Record online: 21 MAY 2003 | DOI: 10.1111/1468-2370.00059.

[6] B. Kosko, Fuzzy Engineering. Prentice Hall, Upper Saddle River, New Jersey, 1997.

[7] A. Minzoni and E. Mounoud, "Rethinking operating models for intangible services: from a mechanistic structure to a sustainable model", Proceedings of the 20th International Conference on Engineering Design (ICED15), Vol. 3, pp. 408-417, 2015.

[8] V. A. Niskanen, "Concept map approach to approximate reasoning with fuzzy extended logic", in M. Fedrizzi, M. Collan and J. Kacprzyk (Eds): Fuzzy technology: present applications and future technology. Studies in Fuzziness and Soft Computing, Vol. 335, pp. 47-70, Springer, 2016.

[9] P. O'Neil and D. Crane, "Policy and Organizational Change in the Federal Aviation Administration: The Ontogenesis of a High-Reliability Organization“, PAR,Vol. 72, Issue 1, pp. 98-111, 2012.

[10] K. S. Park and S.H. Kim, "Fuzzy cognitive maps considering time relationships.", International Journal of Human-Computer Studies, Vol. 42, Issue: 2, pp. 157-168, 1995.

[11] W. Pedrycz, A. Jastrzebska and W. Homenda, "Design of Fuzzy Cognitive Maps for Modeling Time Series", IEEE Transactions of Fuzzy Systems, Vol. 24 (1), pp. 120-130, 2016.

[12] L. Perlow, G. Okhuysen and N. Repenning, "THE SPEED TRAP: Exploring the Relationship Between Decision Making and Temporal Context", Academy of Management Journal, 5: pp. 931 - 955, 2002.

[13] J. Reinecke and Shaz Ansari, "When Times Collide: Temporal Brokerage at the Intersection of Markets and Developments", Academy of Management April 1, vol. 58 nr. 2, pp. 618-648, 2015.

[14] C. Stylios and P. Groumpos, "Modeling Complex Systems Using Fuzzy Cognitive Maps", IEEE Transactions on Systems, Man and Cybernetics Part A 34 (1), pp. 155-162, 2004

[15] F. Taylor, The Principles of Scientific Management. Norton Library 1967, Harper \& Row, Publishers, Incorporated, 1911.

[16] N. Weick and K, Sutcliffe, Managing the unexpected: Resilient performance in an age of uncertainty. San Francisco, CA: Jossey Bass, 2007.

[17] S. Zaheer, A. Stuart and A. Zaheer, "Time Scales and Organizational Theory", The Academy of Management Review Vol. 24, Nr. 4, pp. 725 741, Oct. 1999.

[18] M. Hagiwara, "Extended fuzzy cognitive maps," Proc. of the IEEE Int. Conf. on Fuzzy Systems, pp. 795-801, 1992.

[19] M. Hatwagner, V. Niskanen and L. Koczy, "Behavioral Analysis of Fuzzy Cognitive Map Models by Simulation”, Proc. of IFSA '17 Congress, forthcoming.

[20] K. Lee and S. H. Kwon, "Learning Rule for Time Delay in Fuzzy Cognitive Maps", IEICE Trans. Inf. \& Syst., Vol. E93-D, Nr. 11, pp. 3153-3157, 2010.

[21] W. Stach, L. Kurgan and W. Pedrycz, "Higher-order Fuzzy Cognitive Maps”, Proc. of NAFIPS '06, pp. 166-171, 2006.

[22] H. Zhong, C. Miao, Z. Shen and Y. Feng, "Temporal fuzzy cognitive maps", Proc. of FUZZ-IEEE '08, pp. 1831-1840, 2008. 
[23] H. Zhuge, and X.-F. Luo, "Knowledge map: mathematical model and dynamic behaviors", J. of Computer Science and Technology, vol. 20, no. 3, pp.289-295, 2005.

[24] P. Baranyi, A. Csapo and G. Sallai, Cognitive Infocommunications (CogInfoCom), Springer, 2015. 